Thorax, 1979, 34, 688-689

\title{
Primary meningococcal pericarditis complicated by acute renal failure
}

\author{
R H JONES, H SHUHAIBER, C NOBLE, J KEATS, AND V PARSONS \\ From the Renal and Cardiothoracic Units and Department of Medical Microbiology, \\ King's College Hospital and Medical School, London, UK
}

Purulent pericarditis due to Neisseria meningitidis without meningeal infection is rare, only ten cases having been reported (Solheim et al, 1977; Thompson et $a l, 1977)$. This case is of additional interest because the patient developed and recovered from acute renal failure after surgical drainage of a pericardial effusion causing tamponade.

\section{Case history}

A 33-year-old woman presented with severe retrosternal chest pain and dyspnoea after a one-week period of non-specific malaise without meningitic symptoms. There was no relevant medical history.

The only abnormal findings and investigations on admission were a temperature of $38.4^{\circ} \mathrm{C}$ and white cell count of $25.9 \times 10^{\circ} / 1(87 \%$ neutrophils). The pain radiated to the right hypochondrium, and the next day she became shocked and was transferred to this hospital with a presumptive diagnosis of pulmonary embolus.

On arrival her systolic blood pressure was $70 \mathrm{mmHg}$. Her neck veins were distended and there was tenderness and rigidity in the right hypochondrium; the heart sounds were normal. Chest radiography showed a prominent right pulmonary artery but the electrocardiogram was not diagnostic. An echocardiogram showed pericardial fluid. It seemed unlikely that she would tolerate invasive investigations, and she underwent emergency sternotomy with a view to pulmonary embolectomy. The operative findings were of pericardial tamponade, with $400 \mathrm{ml}$ of pus and fibrinous exudate in the pericardium. This was evacuated and a pericardial drain inserted. There was a short episode of ventricular fibrillation while the chest was being closed but resuscitation was performed rapidly. Postoperative blood pressure was $80 / 60 \mathrm{mmHg}$, and urine output exceeded $150 \mathrm{ml}$ /hour for six hours. Antibiotic treatment with gentamicin, benzylpenicillin, and flucloxacillin was started, and mechanical ventilation with $7 \mathrm{~cm}$ of positive end-expiratory pressure was carried out.

By the next day the patient had developed oliguric acute renal failure, and haemodialysis was performed on the third day after operation. There was heavy proteinuria $(4.35 \mathrm{~g} / \mathrm{l})$, and the urinary sodium concentration was $52.5 \mathrm{mmol} / \mathrm{l}$; the urine contained red blood cells but no casts.

For the next three days coagulation studies were consistent with disseminated intravascular coagulation, which was treated with fresh frozen plasma, small doses of vitamin $K$, and the heparin (2000-3000 IU) needed for haemodialysis.

Tracheostomy was performed on the sixth postoperative day; the patient was haemodynamically stable, an echocardiogram showed a small amount of pericardial fluid, and lung function was satisfactory after withdrawal of positive end-expiratory pressure. The infecting organism was characterised as $N$ meningitidis group W135, and treatment with benzylpenicillin alone was continued. Lumbar puncture was performed; the cerebrospinal fluid was normal.

She was extubated on the 12th postoperative day but needed haemodialysis for a further two weeks. At that time the echocardiogram showed a small amount of fluid in the pericardium, and the electrocardiogram showed changes consistent with myocarditis.

Percutaneous renal biopsy was performed six weeks after her operation. Seven weeks later she was well, with normal renal function but mild hypertension.

\section{Investigations}

Microbiology-The pericardial pus contained Gramnegative intracellular diplococci, and the culture was positive at 18 hours. Countercurrent immunoelectrophoresis was negative. $N$ meningitidis was identified by sugar fermentation and shown to be group W135 by the reference laboratory. Blood cultures, nasopharyngeal swabs, and sputum were sterile, but $N$ meningitidis was isolated from bronchial aspirate one week later. The minimum inhibitory concentration of benzylpenicillin for the $N$ meningitidis isolate was $0.02 \mu \mathrm{g} / \mathrm{ml}$ and was exceeded during treatment.

Immunology-Complement levels were persistently low: $C_{3} 0.33-0.50 \mathrm{~g} / 1$ and $C_{4} 0.05-0.06 \mathrm{~g} / 1$ between the third and fifth postoperative days with $\mathrm{C}_{3}$ still reduced after a further ten days $(0 \cdot 41 \mathrm{~g} / 1)$. Circulating immune complexes were not sought.

Renal biopsy-Light microscopy, immunofluorescence, and electron microscopy showed no significant abnormality.

\section{Discussion}

Primary meningococcal pericarditis is rare; this is the 11 th case to be described and the first in which acute renal failure developed.

About $5 \%$ of meningococcal meningeal infections are complicated by pericarditis (Rubin and Moellering, 
1975). When this occurs late in the course of a meningeal infection, often associated with arthritis, cutaneous vasculitis, episcleritis, panophthalmitis, or myocarditis, it may be due to hypersensitivity to the organism or to endotoxin. Meningococcal sepsis accounts for only $4 \%$ of all cases of purulent pericarditis (Boyle et al, 1961), a condition with a mortality of up to $77 \%$ (Rubin and Moellering, 1975). Vigorous and prolonged antibiotic treatment combined with pericardiocentesis is advocated (Solheim et al, 1977) without the need for intrapericardial antibiotics or open drainage. In the present case thoracotomy was performed because of the possibility of pulmonary embolus and the patient's grave clinical state.

The non-specific malaise and preceding abdominal symptoms experienced by this patient may represent a period of chronic meningococcal septicaemia, although she had no skin or joint lesions. The only "hypersensitivity" complication that developed was myocarditis, with electrocardiographic changes evident four weeks after operation.

The acute renal failure may have been due to tubular necrosis after prolonged hypotension and may have been exacerbated by disseminated intravascular coagulation. Persistent hypocomplementaemia and a report of acute postmeningococcal glomerulonephritis (Rainford et al, 1978) prompted us to seek histological evidence of a glomerular lesion, but the biopsy results did not support this possibility, although the subsequent development of mild hypertension in this patient remains unexplained.
We thank the nursing staff of the intensive care and renal units at King's College Hospital for their invaluable assistance in the care of this patient and Dr J D Abbott at the Manchester Regional Public Health Laboratory, Withington Hospital, for his expert help.

\section{References}

Boyle, J D, Pearce, M L, and Guze, L B (1961). Purulent pericarditis; review of literature and report of eleven cases. Medicine (Baltimore), 40, 119-144.

Rainford, D J, Woodrow, D F, Sloper, J C, de Wardener, H E, and Griffiths, I (1978). Postmeningococcal acute glomerular nephritis. Clinical Nephrology, 9, 249-253.

Rubin, $\mathbf{R} \mathbf{H}$ and Moellering, R C (1975). Clinical, microbiological and therapeutic aspects of purulent pericarditis. American Journal of Medicine, 59, 68-78.

Solheim, K, Molnar, I, Aandahl, M, and Wilhelmsen, $T$ (1977). Primary purulent meningococcal pericarditis with tamponade. Acta Medica Scandinavica, 201, 587-588.

Thompson, D G, Pease, C T, and Howard, A J (1977). Meningococcal pericarditis without meningitis. British Medical Journal, 2, 1330-1331.

Requests for reprints to: Dr R H Jones, Renal Unit, King's College Hospital and Medical School, Denmark Hill, London SE5 9RS. 